\title{
A MIN-MAX THEOREM AND ITS APPLICATIONS TO NONCONSERVATIVE SYSTEMS
}

\section{WEIGUO and LI HONGJIE}

Received 2 August 2001 and in revised form 4 February 2002

\begin{abstract}
A nonvariational generation of a min-max principle by A. Lazer is made. And it is used to prove a new existence results for a nonconservative systems of ordinary differential equations with resonance.
\end{abstract}

2000 Mathematics Subject Classification: 34C25, 49J35.

1. Introduction and lemmas. Let $X$ and $Y$ be two subspaces of a real Hilbert space $H$ such that $H=X \oplus Y$. Let $f: H \rightarrow \mathbb{R}$ be of class $C^{2}$ and denote by $\nabla f$ and $\nabla^{2} f$ the gradient and the Hessian of $f$, respectively. In 1975, Lazer et al. [3] under the following conditions:

$$
\begin{aligned}
& \left\langle\nabla^{2} f(u) h, h\right\rangle \leq-m_{1}\|h\|^{2}, \quad m_{1}>0, \forall h \in X, \forall u \in H ; \\
& \left\langle\nabla^{2} f(u) k, k\right\rangle \geq m_{2}\|k\|^{2}, \quad m_{2}>0, \forall k \in Y, \forall u \in H
\end{aligned}
$$

proved that $f$ has a unique critical point, that is, there exists a unique $v_{0} \in H$ such that $\nabla f\left(v_{0}\right)=0$. Moreover, this critical point is characterized by the equality

$$
f\left(v_{0}\right)=\max _{x \in X} \min _{y \in Y} f(x+y) .
$$

In [5], with the following conditions:

$$
\begin{aligned}
& \left\langle\nabla^{2} f(x+y) h, h\right\rangle \leq-\beta(\|x\|)\|h\|^{2}, \quad \int_{1}^{\infty} \beta(s) d s=\infty, \quad \forall x, h \in X, \forall y \in Y ; \\
& \left\langle\nabla^{2} f(x+y) k, k\right\rangle \geq \alpha(\|y\|)\|k\|^{2}, \quad \int_{1}^{\infty} \alpha(s) d s=\infty, \quad \forall x \in X, \forall y, k \in Y,
\end{aligned}
$$

where $\alpha(s)$ and $\beta(s)$ are two continuous nonincreasing functions from $[0, \infty)$ to $(0, \infty)$, it is proved that $f$ has a unique critical point $v_{0}$ such that $f\left(v_{0}\right)=$ $\max _{x \in X} \min _{y \in Y} f(x+y)$. These results were generalized in [6] and especially for a nonselfadjoint extension of the results of Lazer. This extension was applied in [6] to prove that if the following conditions hold:

$$
N^{2}<\gamma_{1} \leq \gamma_{2}<(N+1)^{2}, \quad \gamma_{1} I \leq \nabla^{2} G(u) \leq \gamma_{2} I,
$$


where $N$ is a nonnegative integer and $I$ is an $n \times n$ matrix, then the following differential equations system has a unique $2 \pi$-periodic solution:

$$
u^{\prime \prime}(t)+A u^{\prime}(t)+\nabla G(u)=e(t)
$$

where $A$ is a constant symmetric matrix. System (1.5) is included in the following nonconservative system (1.6), and assume the following:

$$
u^{\prime \prime}(t)+A u^{\prime}(t)+\nabla G(u, t)=e(t) .
$$

With the use of a nonvariational version of a max-min principle inspired by $[5,6]$, in Section 2 we generalize these unique existence results of system (1.6) to a more general case. To be more precise, we apply a min-max lemma to the periodic boundary value problem of the nonconservative system (1.6) and assume that the following conditions hold:

$$
\begin{gathered}
B_{1}+\alpha(\|u\|) I \leq \nabla^{2} G(u, t) \leq B_{2}-\beta(\|u\|) I, \\
\int_{1}^{+\infty} \min \{\alpha(s), \beta(s)\} d s=+\infty,
\end{gathered}
$$

where $u \in R^{n}, B_{1}$ and $B_{2}$ are two real symmetric matrices, and the eigenvalues of $B_{1}$ and $B_{2}$ are $N_{i}^{2}$ and $\left(N_{i}+1\right)^{2}, i=1, \ldots, n$, respectively; here, $N_{i}, i=1, \ldots, n$ are nonnegative integers and $\alpha(s)$ and $\beta(s)$ are two positive nonincreasing functions for $s \in[0, \infty)$.

In Section 3, we show with some examples that our main results extend the results known so far.

We firstly employ the following lemma from [9].

Lemma 1.1 (see [9]). Assume that $H$ is a Hilbert space. Let $T \in C^{1}(H, H)$, $T^{\prime}(u) \in \operatorname{Isom}(H ; H)$, for all $u \in H$. Then, $T$ is a global diffeomorphism onto $H$ if there exists a continuous map $\omega: R_{+} \rightarrow R_{+} \backslash\{0\}$ such that

$$
\int_{1}^{+\infty} \frac{d s}{\omega(s)}=+\infty, \quad\left\|T^{\prime}(u)^{-1}\right\| \leq \omega(\|u\|)
$$

With this lemma, we can prove the following lemma.

LEMMA 1.2 (see [4]). Let $X$ and $Y$ be two closed subspaces of a real Hilbert space $H$, and $H=X \oplus Y$. Suppose that $T: H \rightarrow H$ is a $C^{1}$-mapping. If there exist two continuous functions $\alpha:[0, \infty) \rightarrow(0, \infty)$ and $\beta:[0, \infty) \rightarrow(0, \infty)$ such that

$$
\begin{aligned}
\left\langle T^{\prime}(u) x, x\right\rangle & \leq-\alpha(\|u\|)\|x\|^{2}, \\
\left\langle T^{\prime}(u) y, y\right\rangle & \geq \beta(\|u\|)\|y\|^{2}, \\
\left\langle T^{\prime}(u) x, y\right\rangle & =\left\langle x, T^{\prime}(u) y\right\rangle
\end{aligned}
$$


for arbitrary $u \in H, x \in X, y \in Y$, and

$$
\int_{1}^{+\infty} \min \{\alpha(s), \beta(s)\} d s=+\infty
$$

then $T$ is a diffeomorphism from $H$ onto $H$.

The following lemma is required in the proof of Theorem 2.2.

LEMMA 1.3 (see [2]). Let $H$ be a vector space such that for subspaces $Y$ and $Z, H=Z \oplus Y$. If $Z$ is finite dimensional and $X$ is a subspace of $H$ such that $X \cap Y=\{0\}$ and dimension $X=\operatorname{dimension} Z$, then $H=X \oplus Y$.

2. Unique existence. Assume that $G(u, t)$ is continuous for $(u, t) \in R^{n} \times$ $[0,2 \pi]$ and twice continuously differiable about $u$. Denote by $\nabla G(u, t)$ and $\nabla^{2} G(u, t)$ the gradient and the Hessian of $G(u, t)$, respectively. We will investigate the unique existence of periodic solutions for system (1.6).

Firstly, we introduce the following definition.

DEFINITION 2.1. The real symmetric matrix $A$ is called admissible with two real symmetric matrices $B_{1}$ and $B_{2}$ if there exist orthogonal matrices $P_{1}$ and $P_{2}$ such that $P_{1}^{T} B_{1} P_{1}, P_{2}^{T} B_{2} P_{2}$, and $P_{1}^{T} A P_{2}$ are simultaneously diagonal matrices.

THEOREM 2.2. If conditions (1.7) hold for all $t \in[0,2 \pi]$, all $u \in R^{n}$, and for $A$ is admissible with the matrices $B_{1}$ and $B_{2}$, then there exists a unique $2 \pi$-periodic solution to system (1.6).

Proof. Because $A$ is admissible with the matrices $B_{1}$ and $B_{2}$, and conditions (1.7) hold for all $t \in[0,2 \pi]$ and all $u \in R^{n}$, we can get orthogonal matrices $P_{1}=\left(a_{1}, a_{2}, \ldots, a_{n}\right), P_{2}=\left(b_{1}, b_{2}, \ldots, b_{n}\right), P_{1}^{T} B_{1} P_{1}=\operatorname{diag}\left(N_{1}^{2}, \ldots, N_{n}^{2}\right), P_{2}^{T} B_{2} P_{2}=$ $\operatorname{diag}\left(\left(N_{1}+1\right)^{2}, \ldots,\left(N_{n}+1\right)^{2}\right)$, and $P_{1}^{T} A P_{2}=\operatorname{diag}\left(\gamma_{1}, \gamma_{2}, \ldots, \gamma_{n}\right)$. Clearly, $a_{i}$ and $b_{i}$ are the eigenvectors of $B_{1}$ and $B_{2}$, respectively, corresponding to the eigenvalues $N_{i}^{2}$ and $\left(N_{i}+1\right)^{2}$, which satisfy

$$
a_{i}^{T} a_{j}=b_{i}^{T} b_{j}=\delta_{i j}, \quad i, j=1,2, \ldots, n,
$$

where $\delta_{i j}=0, i \neq j ; \delta_{i j}=1, i=j$. Define

$$
\begin{gathered}
V=\left\{v(t)=\left(v_{1}(t), \ldots, v_{n}(t)\right)^{T} \mid v_{i}(0)=v_{i}(2 \pi), i=0,1, \ldots, n ;\right. \\
\left.v(t) \text { absolutely continuous and } v^{\prime}(t) \in L^{2}[0,2 \pi]\right\},
\end{gathered}
$$

and it is easy to see that $V$ is a Hilbert space with the following inner product:

$$
\langle u, v\rangle=\int_{0}^{2 \pi}\left[u^{\prime T}(t) v^{\prime}(t)+u^{T}(t) v(t)\right] d t
$$


Denote by $\|\cdot\|_{V}$ the norm induced by this inner product, and define subspaces of $V$ as follows:

$$
\begin{aligned}
& X=\left\{x(t)=\sum_{i=1}^{n} f_{i}(t) a_{i} \mid f_{i}(t)=c_{i 0}+\sum_{m=1}^{N_{i}}\left(c_{i m} \cos m t+d_{i m} \sin m t\right)\right\} \\
& Y=\left\{y(t)=\sum_{i=1}^{n} g_{i}(t) b_{i} \mid g_{i}(t)=\sum_{m=N_{i}+1}^{\infty}\left(p_{i m} \cos m t+q_{i m} \sin m t\right)\right\} \\
& Z=\left\{z(t)=\sum_{i=1}^{n} h_{i}(t) b_{i} \mid h_{i}(t)=p_{i 0}+\sum_{m=1}^{N_{i}}\left(p_{i m} \cos m t+q_{i m} \sin m t\right)\right\},
\end{aligned}
$$

where $N_{i}, i=1, \ldots, n$ are as in (1.7) and $c_{i m}, d_{i m}, p_{i m}$, and $q_{i m}$ are constants. Obviously, $V=Z \oplus Y$. Using the Riesz representation theorem, define a mapping $T: V \rightarrow V$ by

$$
\langle T(u), v\rangle=\int_{0}^{2 \pi}\left[u^{\prime T}(t) v^{\prime}(t)-v^{T}(t) A u^{\prime}(t)-v^{T}(t) \nabla G(u(t), t)\right] d t
$$

for arbitrary $v \in V$. We observe that $T$ is defined implicitly. From (2.5) and the fact that $G$ is $C^{2}$, it can be proved that $T$ is a $C^{1}$-mapping and that

$$
\left\langle T^{\prime}(u) w, v\right\rangle=\int_{0}^{2 \pi}\left[w^{\prime T} v^{\prime}(t)-v^{T}(t) A w^{\prime T}(t)-v^{T}(t) \nabla^{2} G(u, t) w(t)\right] d t
$$

for all $v(t), u(t), w(t) \in V$. Again, from the Riesz representation theorem, there exists an element $d \in V$ satisfying

$$
\langle d, v\rangle=-\int_{0}^{2 \pi} v^{T}(t) e(t) d t
$$

It can be proved that $u$ is a $2 \pi$-periodic solution to (1.6) if and only if $u$ satisfies the operator equation

$$
T(u)=d
$$

We will next show that $T$ satisfies the conditions of Lemma 1.2. This will, in turn, imply that (1.6) has a unique $2 \pi$-periodic solution. For any $x \in X$ and $u \in V$, we have that

$$
\left\langle T^{\prime}(u) x, x\right\rangle=\int_{0}^{2 \pi}\left[x^{\prime T}(t) x^{\prime}(t)-x^{T}(t) A x^{\prime}(t)-x^{T}(t) \nabla^{2} G(u, t) x(t)\right] d t,
$$

where

$$
\begin{aligned}
& \int_{0}^{2 \pi} x^{\prime T}(t) x^{\prime}(t) d t=\int_{0}^{2 \pi} \sum_{i=1}^{n} f_{i}^{\prime 2}(t) d t \leq \sum_{i=1}^{n} N_{i}^{2} \int_{0}^{2 \pi} f_{i}^{2}(t) d t \\
& \int_{0}^{2 \pi} x^{T}(t) A x^{\prime}(t) d t=\left.\frac{1}{2} x^{T}(t) A x(t)\right|_{0} ^{2 \pi}=0
\end{aligned}
$$


By (1.7), we have

$$
\begin{aligned}
\int_{0}^{2 \pi} x^{T}(t) \nabla^{2} G(u, t) x(t) d t \\
\quad \geq \int_{0}^{2 \pi} x^{T}(t) B_{1} x(t) d t+\alpha\left(\|u\|_{V}\right) \int_{0}^{2 \pi} x^{T}(t) x(t) d t \\
\quad=\int_{0}^{2 \pi} \sum_{i=1}^{n} \sum_{j=1}^{n} f_{i}(t) f_{j}(t) a_{i}^{T} B_{1} a_{j} d t+\alpha\left(\|u\|_{V}\right) \int_{0}^{2 \pi} x^{T}(t) x(t) d t \\
\quad=\sum_{i=1}^{n} N_{i}^{2} \int_{0}^{2 \pi} f_{i}^{2}(t) d t+\alpha\left(\|u\|_{V}\right) \int_{0}^{2 \pi} x^{T}(t) x(t) d t \\
\|x\|_{V}^{2}=\int_{0}^{2 \pi} x^{T}(t) x(t) d t+\int_{0}^{2 \pi} x^{\prime T}(t) x^{\prime}(t) d t \\
\leq\left(M^{2}+1\right) \int_{0}^{2 \pi} x^{T}(t) x(t) d t
\end{aligned}
$$

where $M=\max _{1 \leq i \leq n}\left\{N_{i}\right\}$, therefore

$$
\left\langle T^{\prime}(u) x, x\right\rangle \leq-\frac{\alpha\left(\|u\|_{V}\right)}{M^{2}+1}\|x\|_{V}^{2}
$$

Similarly, from

$$
\begin{aligned}
\int_{0}^{2 \pi} y^{\prime T}(t) y^{\prime}(t) d t \geq & \sum_{i=1}^{n}\left(N_{i}+1\right)^{2} \int_{0}^{2 \pi} g_{i}^{2}(t) d t \\
-\int_{0}^{2 \pi} y^{T}(t) \nabla^{2} G(u, t) y(t) d t \geq & -\int_{0}^{2 \pi} y^{T}(t) B_{2} y(t) d t \\
& +\beta\left(\|u\|_{V}\right) \int_{0}^{2 \pi} y^{T}(t) y(t) d t
\end{aligned}
$$

we can get that for all $y \in Y$ and all $u \in V$,

$$
\begin{aligned}
\int_{0}^{2 \pi} & \left\{\left[1+(M+1)^{2}\right]\left[y^{\prime T}(t) y^{\prime}(t)-y^{T}(t) \nabla^{2} G(u, t) y(t)\right]\right. \\
& \left.-\beta\left(\|u\|_{V}\right)\left[y^{\prime T} y^{\prime}(t)+y^{T}(t) y(t)\right]\right\} d t \\
= & {\left[1+(M+1)^{2}-\beta\left(\|u\|_{V}\right)\right] \int_{0}^{2 \pi} y^{\prime T}(t) y^{\prime}(t) d t } \\
& -\int_{0}^{2 \pi}\left[1+(M+1)^{2}\right] y^{T}(t) \nabla^{2} G(u, t) y(t) d t-\beta\left(\|u\|_{V}\right) \int_{0}^{2 \pi} y^{T}(t) y(t) d t \\
\geq & {\left[1+(M+1)^{2}-\beta\left(\|u\|_{V}\right)\right] \sum_{i=1}^{n}\left(N_{i}+1\right)^{2} \int_{0}^{2 \pi} g_{i}^{2}(t) d t } \\
& -\int_{0}^{2 \pi}\left[1+(M+1)^{2}\right] y^{T}(t) B_{2} y(t) d t+(M+1)^{2} \beta\left(\|u\|_{V}\right) \int_{0}^{2 \pi} y^{T}(t) y(t) d t
\end{aligned}
$$




$$
\begin{aligned}
= & {\left[1+(M+1)^{2}-\beta\left(\|u\|_{V}\right)\right] \sum_{i=1}^{n}\left(N_{i}+1\right)^{2} \int_{0}^{2 \pi} g_{i}^{2}(t) d t } \\
& -\left[1+(M+1)^{2}\right] \sum_{i=1}^{n}\left(N_{i}+1\right)^{2} \int_{0}^{2 \pi} g_{i}^{2}(t) d t+(M+1)^{2} \beta\left(\|u\|_{V}\right) \sum_{i=1}^{n} \int_{0}^{2 \pi} g_{i}^{2}(t) d t \\
= & \beta\left(\|u\|_{V}\right) \sum_{i=1}^{n}\left[(M+1)^{2}-\left(N_{i}+1\right)^{2}\right] \int_{0}^{2 \pi} g_{i}^{2}(t) d t \geq 0,
\end{aligned}
$$

and from

$$
\int_{0}^{2 \pi} y^{T}(t) A y^{\prime}(t) d t=\left.\frac{1}{2} y^{T}(t) A y(t)\right|_{0} ^{2 \pi}=0,
$$

we can prove that for all $y \in Y$ and all $u \in V$,

$$
\begin{aligned}
\left\langle T^{\prime}(u) y, y\right\rangle & =\int_{0}^{2 \pi}\left[y^{\prime T}(t) y^{\prime}(t)-y^{T}(t) A y^{\prime}(t)-y^{T}(t) \nabla^{2} G(u, t) y(t)\right] d t \\
& \geq \frac{\beta\left(\|u\|_{V}\right)}{(M+1)^{2}+1}\|y\|_{V}^{2} .
\end{aligned}
$$

Obviously, for all $x \in X$ and all $y \in Y$, we have the following:

$$
\begin{aligned}
& \left\langle T^{\prime}(u) x, y\right\rangle-\left\langle x, T^{\prime}(u) y\right\rangle \\
& \quad=\int_{0}^{2 \pi}\left[x^{T}(t) A y^{\prime}(t)-y^{T}(t) A x^{\prime}(t)\right] d t \\
& \quad=\int_{0}^{2 \pi} 2\left(f_{1}(t), \ldots, f_{n}(t)\right) P_{1}^{T} A P_{2}\left(g_{1}(t), \ldots, g_{n}(t)\right)^{T} d t \\
& \quad=\int_{0}^{2 \pi} \sum_{i=1}^{n} 2 \gamma_{i} f_{i}(t) g_{i}^{\prime}(t) d t=0 .
\end{aligned}
$$

Let $\alpha_{1}=\alpha(s) /\left(M^{2}+1\right)$ and $\beta_{1}(s)=\beta(s) /\left((M+1)^{2}+1\right)$, then

$$
c(s)=\min \left\{\alpha_{1}(s), \beta_{1}(s)\right\} \geq \min \{\alpha(s), \beta(s)\} /\left((M+1)^{2}+1\right) .
$$

Based on conditions (1.7), $\int_{1}^{+\infty} c(s) d s=+\infty$. Since $T^{\prime}(u)$ is positive definite on $Y$ and negative definite on $X$, we see that $X \cap Y=\{0\}$. Moreover, it is readily seen that

$$
\text { dimension } X=\operatorname{dimension} Z=\sum_{i=1}^{n}\left(2 N_{i}+1\right) .
$$

Thus, since it was shown above that $V=Z \oplus Y$, it follows, by application of Lemma 1.3, that $V=X \oplus Y$. We may, therefore, apply Lemma 1.2 to get the conclusion of the theorem. 
If we set $V=\left\{v(t)=\left(v_{1}(t), \ldots, v_{n}(t)\right)^{T} \mid v_{i}(0)=v_{i}(\pi)=0, i=1, \ldots, n ; v(t)\right.$ to be absolutely continuous and $\left.v^{\prime}(t) \in L^{2}[0, \pi]\right\}$, it is easy to know that $V$ is a Hilbert space about the following inner product:

$$
\langle u, v\rangle=\int_{0}^{\pi}\left[u^{\prime T}(t) v^{\prime}(t)+u^{T}(t) v(t)\right] d t .
$$

Again, define the norm induced by this inner product and subspaces $X, Y$, and $Z$, correspondingly; we can prove the following theorem similarly.

THEOREM 2.3. Assume that $G(u, t)$ is continuous and $C^{2}$-mapping with respect to $u$ and that conditions (1.7) hold for all $t \in[0, \pi]$, all $u \in R^{n}$, and for $A$ is admissible with matrices $B_{1}$ and $B_{2}$. Let $e(t)$ be a continuous function. Then, there exists a unique solution to (1.6), which satisfies boundary value condition $u(0)=u(\pi)=0$.

Especially, when $B_{1}=N^{2} I$ and $B_{2}=(N+1)^{2} I$, where $N$ is natrual and $I$ is $n \times n$ identity matrix, $A$ is admissible with $B_{1}$ and $B_{2}$ as long as $A$ is real symmetric. So, we have the following corollary.

COROLLARY 2.4. Assume that $A$ is real symmetric and there exist two positive continuous functions $\delta_{1}$ and $\delta_{2}: R^{n} \rightarrow \mathbb{R}$ such that for all $u \in R^{n}$ and all $t \in$ $[0,2 \pi]$

$$
N^{2} I<\delta_{1}(u) I \leq \nabla^{2} G(u, t) \leq \delta_{2}(u) I<(N+1)^{2} .
$$

Let $\rho(r)=\min \left\{1-\max _{\sum_{i=1}^{n}\left|\xi_{i}\right| \leq r}\left(\delta_{2}(\xi) /(N+1)^{2}\right), \max _{\sum_{i=1}^{n}\left|\xi_{i}\right| \leq r}\left(\delta_{1}(\xi) / N^{2}\right)-\right.$ $1\}$, and if $\int_{1}^{+\infty} \rho(r) d r=+\infty$, then the system (1.6) has a unique $2 \pi$-periodic solution.

If we set $A=0$, system (1.6) becomes a conservative system and admissibility is trivial. So, the main conclusion in [7] (the method there is different from ours) is a corollary of Theorem 2.2.

COROLLARY 2.5. Assume that there exist integers $N_{i} \geq 0$ such that for all $u \in R^{n}$ and all $t \in[0,2 \pi]$,

$$
\begin{gathered}
N_{i}^{2}<\lambda_{i}(u, t)<\left(N_{i}+1\right)^{2}, \quad i=1, \ldots, n \\
\delta(\|u\|, t)=\max _{\|v\| \leq\|u\|}\left\{\min _{1 \leq i \leq n}\left\{\lambda_{i}(u, t)-N_{i}^{2},\left(N_{i}+1\right)^{2}-\lambda_{i}(u, t)\right\}\right\},
\end{gathered}
$$

where $\lambda_{i}(u, t), i=1,2, \ldots, n$ denote the eigenvalues of $\nabla^{2} G(u)$. If $\int_{1}^{+\infty} \delta(s, t) d s$ $=+\infty$ for all $t \in[0,2 \pi]$, then there exists a $2 \pi$-periodic solution to (1.5).

Let $G(u, t)=G(u)$ and

$$
c(s)=\min \{\alpha(s), \beta(s)\} \geq c_{0}>0
$$

we can get the following unique existence corollary. 
COROLLARY 2.6. If the real symmetric matrix $A$ is admissible with real symmetric matrices $B_{1}$ and $B_{2}$, then assume that

$$
B_{1} \leq \nabla^{2} G(u) \leq B_{2}, \quad N_{i}^{2}<\lambda_{i} \leq \mu_{i}<\left(N_{i}+1\right)^{2},
$$

where $\lambda_{i}$ and $\mu_{i}$ are eigenvalues of $B_{1}$ and $B_{2}$, respectively, and $N_{i}, i=1, \ldots, n$ are nonnegative integers; there exists a unique $2 \pi$-periodic solution to system (1.5).

3. Examples. It should be pointed out that conditions (1.9) and (1.11) are not completely the same as (1.3). In fact, from (1.3), we know that $\alpha(\|x\|)$ depends on subspace $X$ and $\beta(\|y\|)$ depends upon subspace $Y$. So, condition (1.3) is more strict than conditions (1.9). But, from (1.11), we can deduce the following conditions:

$$
\int_{1}^{+\infty} \alpha(s) d s=+\infty, \quad \int_{1}^{+\infty} \beta(s) d s=+\infty .
$$

Conversely, note that (3.1) does not imply (1.11). Now, we give an example to illustrate it.

EXAMPLE 3.1. First of all, we define two nondecreasing functions as follows:

$$
\begin{array}{ll}
\alpha(x)=2^{-(2 i+1)^{2}}, & x_{2 i-1} \leq x<x_{2 i+1} \\
\beta(x)=2^{-(2 i+2)^{2}}, & x_{2 i} \leq x<x_{2 i+2}
\end{array}
$$

where $x_{i}=\sum_{k=0}^{i} 2^{k^{2}}, i=0,1, \ldots, x_{-1}=0$, and $\beta(x)=1$, when $0 \leq x<1$.

It is easy to see that $\alpha(x)$ and $\beta(s)$ are two nondecreasing positive functions for all $x \in[0,+\infty)$; and the number of noncontinuous points is countable infinite. We also have

$$
\begin{aligned}
\int_{1}^{+\infty} \alpha(x) d x & =1+\sum_{i=1}^{+\infty} \frac{x_{2 i+1}-x_{2 i-1}}{2^{(2 i+1)^{2}}} \\
& =1+\sum_{i=1}^{+\infty} \frac{2^{(2 i+1)^{2}}+2^{(2 i)^{2}}}{2^{(2 i+1)^{2}}}=+\infty \\
\int_{1}^{+\infty} \beta(x) d x & =\sum_{i=1}^{+\infty} \frac{x_{2 i}-x_{2 i-2}}{2^{(2 i)^{2}}}=\sum_{i=1}^{+\infty} \frac{2^{(2 i)^{2}}+2^{(2 i-1)^{2}}}{2^{(2 i)^{2}}}=+\infty, \\
\int_{1}^{+\infty} \min \{\alpha(x), \beta(x)\} d x & \leq \sum_{i=0}^{+\infty} \frac{x_{2 i+1}-x_{2 i}}{2^{(2 i+2)^{2}}}+\sum_{i=0}^{+\infty} \frac{x_{2 i}-x_{2 i-1}}{2^{(2 i+1)^{2}}} \\
& \leq \sum_{i=0}^{+\infty} \frac{2^{(2 i+1)^{2}}}{2^{(2 i+2)^{2}}}+\sum_{i=0}^{+\infty} \frac{2^{(2 i)^{2}}}{2^{(2 i+1)^{2}}}<+\infty
\end{aligned}
$$

Secondly, from the definition of $\alpha(x)$ and $\beta(x)$, it is easy to make them continuous and even continuously differentiable, and then they are still positive nondecreasing and satisfy (3.1), but they do not satisfy conditions (1.11). 
We can state, from the following example, that Theorem 2.3 is more general than the results of $[1,2,3,4,5,6,7,8]$.

EXAMPLE 3.2. Assume that $f(t)$ is continuous and $2 \pi$-periodic in (1.6). Let

$$
\begin{aligned}
G(u, t)= & \frac{5}{4}\left(1+\frac{4}{5} \sin ^{2} t\right)\left(u_{1}^{2}+u_{2}^{2}\right)+\frac{3}{2}\left(1+\frac{2}{3} \sin ^{2} t\right) u_{1} u_{2} \\
& +u_{1} \ln \left(u_{1}+\sqrt{1+u_{1}^{2}}\right)+u_{2} \ln \left(u_{2}+\sqrt{1+u_{2}^{2}}\right) \\
& -\sqrt{1+u_{1}^{2}}-\sqrt{1+u_{2}^{2}}+C_{1} u_{1}+C_{2} u_{2}
\end{aligned}
$$

then

$$
\begin{aligned}
& \nabla^{2} G(u, t)=\left(\begin{array}{cc}
\frac{5}{2}\left(1+\frac{4}{5} \sin ^{2} t\right)+\frac{1}{\sqrt{1+u_{1}^{2}}} & \frac{3}{2}\left(1+\frac{2}{3} \sin ^{2}(t)\right) \\
\frac{3}{2}\left(1+\frac{2}{3} \sin ^{2}(t)\right) & \frac{5}{2}\left(1+\frac{4}{5} \sin ^{2} t\right)+\frac{1}{\sqrt{1+u_{2}^{2}}}
\end{array}\right), \\
&\left(\begin{array}{cc}
\frac{5}{2} & \frac{3}{2} \\
\frac{3}{2} & \frac{5}{2}
\end{array}\right)+\left(\begin{array}{cc}
\frac{1}{\sqrt{1+u_{1}^{2}}} & 0 \\
0 & \frac{1}{\sqrt{1+u_{2}^{2}}}
\end{array}\right) \leq \nabla^{2} G(u, t) \\
& \leq\left(\begin{array}{cc}
\frac{13}{2} & \frac{5}{2} \\
\frac{5}{2} & \frac{13}{2}
\end{array}\right)-\left(\begin{array}{cc}
1-\frac{1}{\sqrt{1+u_{1}^{2}}} & 0 \\
0 & 1-\frac{1}{\sqrt{1+u_{2}^{2}}}
\end{array}\right) .
\end{aligned}
$$

It is easy to see that $G(u, t)$ satisfies (1.7). Therefore, there exists a unique $2 \pi$-periodic solution to (1.6) by Theorem 2.2 , but we cannot make this conclusion from $[1,2,3,4,5,6,7,8]$.

\section{REFERENCES}

[1] K. J. Brown and S. S. Lin, Periodically perturbed conservative systems and a global inverse function theorem, Nonlinear Anal. 4 (1980), no. 1, 193-201.

[2] A. C. Lazer, Application of a lemma on bilinear forms to a problem in nonlinear oscillations, Proc. Amer. Math. Soc. 33 (1972), 89-94.

[3] A. C. Lazer, E. M. Landesman, and D. R. Meyers, On saddle point problems in the calculus of variations, the Ritz algorithm, and monotone convergence, J. Math. Anal. Appl. 52 (1975), no. 3, 594-614.

[4] W. Li, Periodic solutions for $2 k$ th order ordinary differential equations with resonance, J. Math. Anal. Appl. 259 (2001), no. 1, 157-167.

[5] R. F. Manasevich, A min max theorem, J. Math. Anal. Appl. 90 (1982), no. 1, 64-71.

[6] _ A nonvariational version of a max-min principle, Nonlinear Anal. 7 (1983), no. 6, 565-570.

[7] Z. H. Shen, On the periodic solution to the Newtonian equation of motion, Nonlinear Anal. 13 (1989), no. 2, 145-149. 
[8] S. A. Tersian, A minimax theorem and applications to nonresonance problems for semilinear equations, Nonlinear Anal. 10 (1986), no. 7, 651-668.

[9] G. Zampieri, Diffeomorphisms with Banach space domains, Nonlinear Anal. 19 (1992), no. 10, 923-932.

Li Weiguo: Department of Applied Mathematics, University of Petroleum, Dongying 257061, Shandong Province, China

E-mail address: 1 iwg@mai 1.hdpu.edu.cn

Li Hongjie: Department of Mathematics, Linyi Teacher's College, Linyi 276005, Shandong Province, China.

E-mail address: 1yjk@263.com 


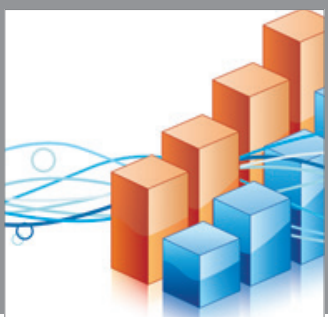

Advances in

Operations Research

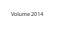

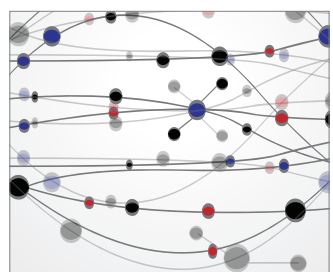

\section{The Scientific} World Journal
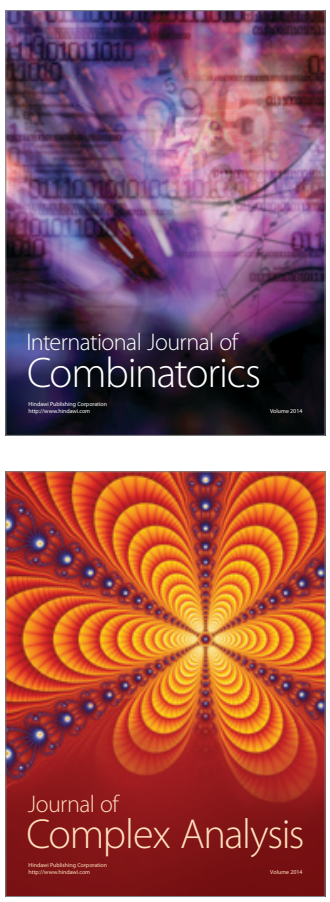

International Journal of

Mathematics and

Mathematical

Sciences
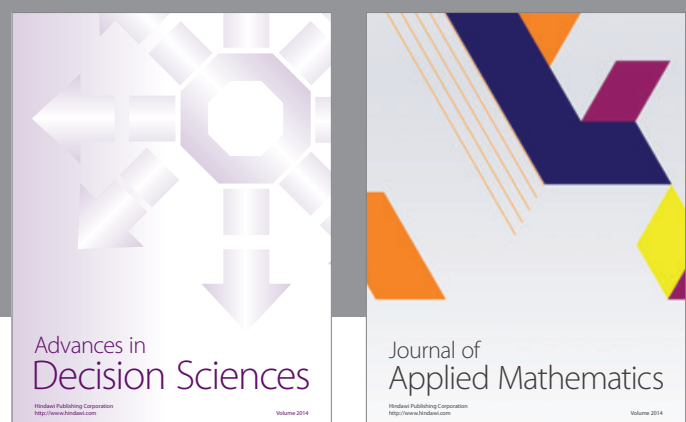

Journal of

Applied Mathematics
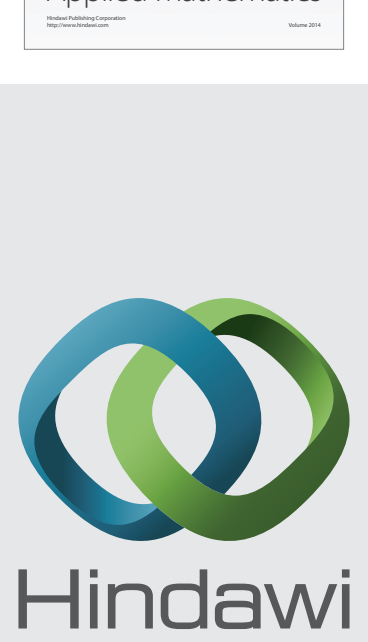

Submit your manuscripts at http://www.hindawi.com
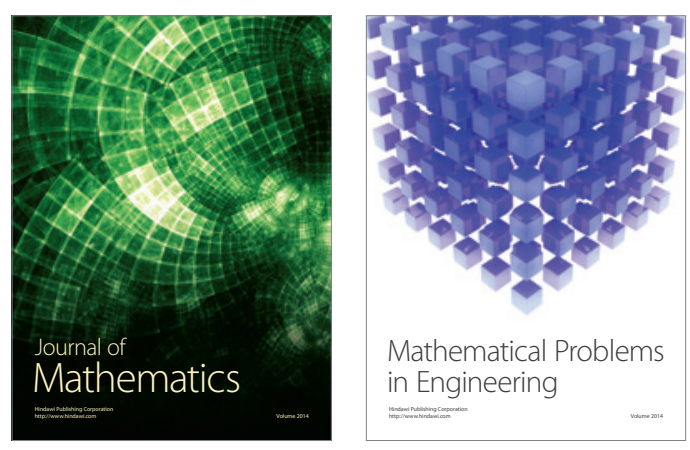

Mathematical Problems in Engineering
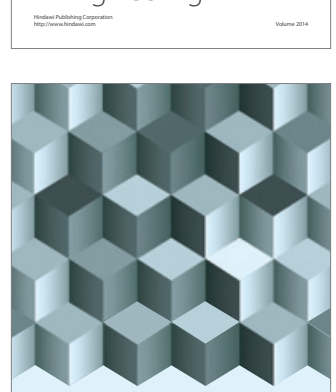

Journal of

Function Spaces
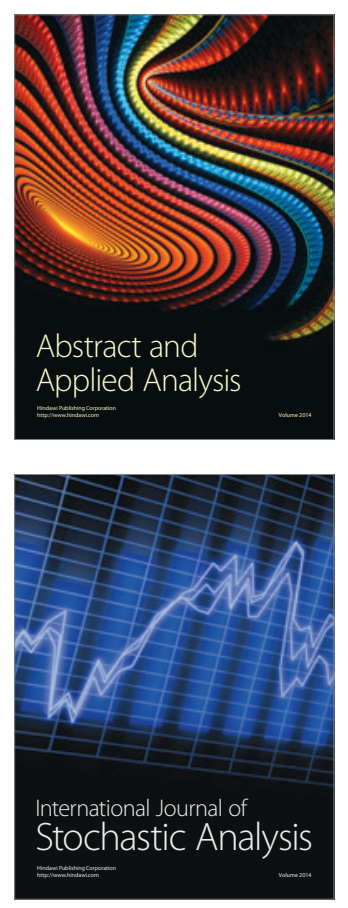

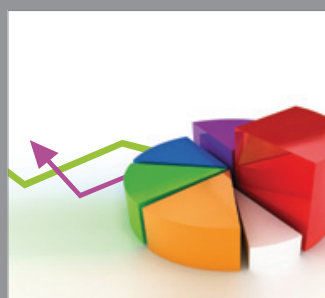

ournal of

Probability and Statistics

Promensencen
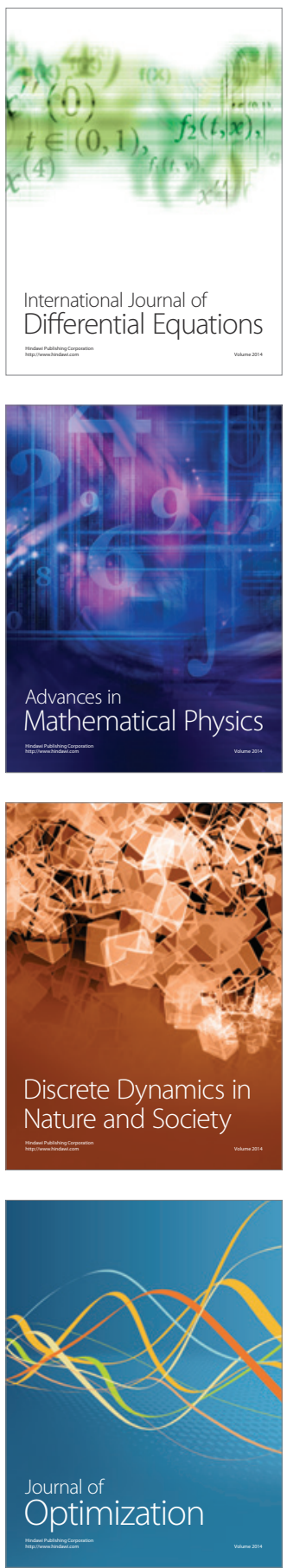